\title{
Mitochondrial DNA sequencing demonstrates clonality of peritoneal implants of borderline ovarian tumors
}

\author{
Giulia Girolimetti ${ }^{1}$, Pierandrea De laco ${ }^{2}$, Martina Procaccini ${ }^{2}$, Riccardo Panzacchi ${ }^{3}$, Ivana Kurelac ${ }^{1}$, \\ Laura Benedetta Amato ${ }^{1}$, Giulia Dondi ${ }^{2}$, Giacomo Caprara ${ }^{4}$, Claudio Ceccarelli ${ }^{5}$, Donatella Santini ${ }^{3}$, \\ Anna Maria Porcelli ${ }^{6}$, Anna Myriam Perrone ${ }^{2+}$ and Giuseppe Gasparre ${ }^{1 *+} \mathbb{B}$
}

\begin{abstract}
Borderline ovarian tumors are rare low malignant potential neoplasms characterized by the absence of stromal invasion, whose main prognostic factors are stage and type of peritoneal implants. The latter are defined as invasive when cell proliferation invades the underlying tissue (peritoneal surface, omentum and intestinal wall), or noninvasive. It is still unknown if these implants are metastatic spread from the primary ovarian mass or a neoplastic transformation de novo of the peritoneal surface. Mitochondrial DNA sequencing was performed to assess clonality in eight patients presenting both borderline ovarian tumors and implants. In 37.5\% of the cases, the same mitochondrial DNA mutation was present in both borderline ovarian tumors and the peritoneal implant, being this evidence that implants may arise as a consequence of a spread from a single ovarian site.
\end{abstract}

Keywords: Gynecological cancer, Mitochondrial DNA mutations, Borderline ovarian tumors, Peritoneal implants

\section{Findings \\ Background}

Borderline ovarian tumors (BOTs) represent a heterogeneous group of noninvasive neoplasms of low malignant potential. They comprise about $15-20 \%$ of all epithelial ovarian malignancies [1] and are usually diagnosed as being limited to the ovary. The 10-year survival rate for women with stage I BOT is around $70 \%$, caused by subsequently recurrent disease or progression to invasive carcinoma [2, 3]. Standard treatment is hysterectomy with bilateral adnexectomy and multiple peritoneal biopsies. In young patients conservative treatment is an option and they may undergo surgery limited to a unilateral salpingo-ovariectomy with multiple biopsies [4]. Serous histotype represents $65 \%$ of all the BOTs [5], about $35 \%$ of which can occur in association with serous lesions involving the peritoneum, i.e. implants, defined

\footnotetext{
* Correspondence: giuseppe.gasparre@gmail.com

${ }^{\dagger}$ Equal contributors

'Department of Medical and Surgical Sciences (DIMEC) - Unit of Medical Genetics, University of Bologna Medical School, Via G. Massarenti 9, 40138 Bologna, Italy

Full list of author information is available at the end of the article
}

as either invasive or noninvasive depending on their microscopic appearance. Invasive implants are found in a lower number of patients (22\%) compared to noninvasive ones (78\%) [5], and the survival rate is around 66\% after a mean follow-up of 7.4 years, compared to $95 \%$ for patients with noninvasive implants [6]. A study on 80 cases of serous BOTs with noninvasive implants showed that after a follow-up of 15 years, $44 \%$ of patients presented recurrences and 25\% died of disease [7]. The pathological stage and sub-classification of extraovarian disease into invasive and noninvasive implants, together with the presence of postoperative macroscopic residual masses, currently appears to be the major predictor not only for recurrence, but also for survival $[8,9]$.

In the last years, clonality studies have attempted to elucidate whether multiple tumor nodules arise as a result of a spread from a single ovarian site or whether such deposits are polyclonal, representing independent primary tumors, with discordant results $[10,11]$.

BOT patients recurrence and survival change substantially depending on whether a peritoneal implant arises or not. Since women with BOT and peritoneal lesions 
usually have a good prognosis, the latter are classified as implants instead of metastases. These masses are considered as an extra-ovarian spread of the primary tumor [11], but several studies highlight their differences with the latter, considering implants as independent masses of polyclonal origin [10]. In spite of this still open dilemma, approaches for discrimination between the monoclonal or polyclonal nature of peritoneal implants are still lacking. Mitochondrial DNA sequencing was recently shown to be a robust tool to define clonality in simultaneously detected tumors of the female genital tract $[12,13]$ as it is virtually impossible that the same tumor-specific mutation may arise in two independent neoplasms. We here apply such technique to BOTs and their implants.

\section{Results}

We performed whole mitochondrial DNA sequencing on eight patients presenting with serous BOTs and implants (Table 1), after collection of informed consent within the frame of the Mitochondria in Progression of Endometrial and Ovarian cancer - MiPEO study, approved by the local ethical committee. An alphanumeric code (from B1 to B8) was assigned to the cases to maintain anonymity.

Three out of eight patients underwent complete staging and $5 / 8(62.5 \%)$ fertility sparing treatment following their wish to become pregnant. Based on histopathological analyses, all samples were serous BOTs, 2 of whom with one small focus of intraepithelial cancer in the ovarian cyst. Noninvasive implants were diagnosed in $7 / 8$ cases $(87.5 \%)$, only one patient presenting with an invasive implant (12.5\%), and relapsing after 30 months from the first surgery. Recurrences were observed only in patients with fertility sparing treatment (4/5-80\%). Three out of seven patients (42.8\%) diagnosed with noninvasive implants presented relapse after their first surgery (Table 1). All patients were alive and free of disease at the latest follow up.

The entire mitochondrial DNA sequence was obtained from all 16 single BOT and implant samples [14] and variants carefully filtered for pathogenicity through MToolBox $[15,16]$. Detailed materials and methods are available as Additional file 1. For all eight patients, DNA extracted from unaffected tissue was used to detect tumor-specific and non-tumor-specific variants. Sequences of B1-B8 samples, including matched nontumor sequences, were submitted to the public human mitochondrial database HmtDB [17] and a list of specimens and HmtDB identifiers is reported in Additional file 2. Overall, 7 tumor-specific variants in coding genes were found in $4 / 8$ patients (50\%) (Table 2).

In patient $\mathrm{B} 1$, the m.15570 $>\mathrm{C} /(M T-C Y B)$ was found in both BOT and implant (Fig. 1a) whereas the m.4810G $>\mathrm{A} /(M T-N D 2)$ was found only in BOT. B2 carried the mutations m.3428G $>\mathrm{A} /(M T-N D 1)$ and m.15219ins $/(M T-C Y B)$ only in BOT. In patient B3, we found the m.11984 $\mathrm{T}>\mathrm{C} /(M T-N D 4)$ in both BOT and implant (Fig. 1b). In patient B4, the m.15449 $\mathrm{T}>\mathrm{C} /(M T$ $C Y B)$ was found in both BOT and implant (Fig. 1c) whereas the $\mathrm{m} .3352 \mathrm{G}>\mathrm{A} /(M T-N D 1)$ was found only in BOT. None of the corresponding matched non-tumor samples was shown to carry these mitochondrial DNA mutations. All mitochondrial DNA mutations found

Table 1 Cases and histopathology

\begin{tabular}{|c|c|c|c|c|c|c|c|c|}
\hline Patients & $\begin{array}{l}\text { Age at } \\
\text { diagnosis }\end{array}$ & Surgery & Histology & $\begin{array}{l}\text { Staging (FIGO } \\
\text { 2014) }\end{array}$ & $\begin{array}{l}\text { Implant } \\
\text { type }\end{array}$ & Implant localization & DFS & OS \\
\hline B1 & 35 & FS & Serous BOT & $\| \mathrm{B}$ & $\begin{array}{l}\text { Non } \\
\text { invasive }\end{array}$ & Pelvic peritoneum & 48 & 74 \\
\hline B2 & 44 & CS & Serous BOT & $\| C 3$ & $\begin{array}{l}\text { Non } \\
\text { invasive }\end{array}$ & Pelvic peritoneum & 34 & 34 \\
\hline B3 & 30 & FS & $\begin{array}{l}\text { Serous BOT with } \\
\text { intraepithelial carcinoma }\end{array}$ & $\| I A$ & $\begin{array}{l}\text { Non } \\
\text { invasive }\end{array}$ & Pelvic peritoneum and omentum & 31 & 31 \\
\hline B4 & 81 & CS & Serous BOT & $\| \mathrm{B}$ & $\begin{array}{l}\text { Non } \\
\text { invasive }\end{array}$ & Pelvic peritoneum & 120 & 120 \\
\hline B5 & 23 & FS & $\begin{array}{l}\text { Serous BOT with } \\
\text { intraepithelial carcinoma }\end{array}$ & $11 \mathrm{C} 3$ & Invasive & Pelvic peritoneum & 30 & 67 \\
\hline B6 & 32 & FS & Serous BOT & IIC3 & $\begin{array}{l}\text { Non } \\
\text { invasive }\end{array}$ & Pelvic peritoneum & 7 & 68 \\
\hline B7 & 34 & FS & Serous BOT & IIIA & $\begin{array}{l}\text { Non } \\
\text { invasive }\end{array}$ & Pelvic peritoneum, pararectal peritoneum & 13 & 128 \\
\hline B8 & 63 & CS & Serous BOT & $\| I A$ & $\begin{array}{l}\text { Non } \\
\text { invasive }\end{array}$ & $\begin{array}{l}\text { Pelvic peritoneum, omentum and right } \\
\text { diaphragmatic peritoneum }\end{array}$ & 16 & 16 \\
\hline
\end{tabular}

Age, surgery, histology and FIGO stage of borderline ovarian tumors, implants type and localization, months from treatment until first relapse and months from diagnosis to last follow up are shown

Abbreviations: FS Fertility Sparing treatment, CS Complete Staging, BOT Borderline Ovarian Tumor, DFS Disease Free Survival, OS Overall Survival 
Table 2 Mitochondrial DNA mutations

\begin{tabular}{|c|c|c|c|c|c|c|c|}
\hline Sample & $\begin{array}{l}\text { Mitochondrial DNA } \\
\text { Mutations }\end{array}$ & $\begin{array}{l}\text { Mitochondrial DNA } \\
\text { mutations localization }\end{array}$ & Mutation type & Amino Acid substitution & Gene & Variability & DS \\
\hline \multirow[t]{2}{*}{ B1 } & $m .4810 G>A$ & BOT & Nonsense & W114X & MT-ND2 & 0.0 & - \\
\hline & $\mathrm{m} .15570 \mathrm{~T}>\mathrm{C}$ & $\mathrm{BOT}+\mathrm{PI}$ & Missense & L275P & MT-CYB & 0.0 & 0.892 \\
\hline \multirow[t]{2}{*}{ B2 } & m.3428G >A & BOT & Missense & G41D & MT-ND1 & 0.0 & 0.909 \\
\hline & m.15219insA & BOT & Frameshift & - & MT-CYB & 0.0 & - \\
\hline B3 & m.11984T >C & $\mathrm{BOT}+\mathrm{Pl}$ & Missense & $\mathrm{Y} 409 \mathrm{H}$ & MT-ND4 & 0.002 & 0.764 \\
\hline \multirow[t]{3}{*}{ B4 } & m.3352G >A & BOT & Missense & $\mathrm{A} 16 \mathrm{~T}$ & MT-ND1 & 0.0 & 0.827 \\
\hline & m.15449T >C & $\mathrm{BOT}+\mathrm{PI}$ & Missense & F235L & $M T-C Y B$ & 0.00642 & 0.088 \\
\hline & $\mathrm{m} .16189 \mathrm{~T}>\mathrm{C}$ & $\mathrm{BOT}+\mathrm{PI}$ & SNP & - & MT-D-loop & 0.767 & - \\
\hline \multirow[t]{2}{*}{ B5 } & m.310insC & BOT & Insertion & - & MT-D-loop & 0.215 & - \\
\hline & m.310delCC & PI & Deletion & - & MT-D-loop & 0.215 & - \\
\hline
\end{tabular}

All mitochondrial DNA mutations reported in the table are tumor-specific and heteroplasmic

Abbreviations: BOT Borderline Ovarian Tumor, PI Peritoneal Implant, SNP Single Nucleotide Polymorphism, DS Disease Score
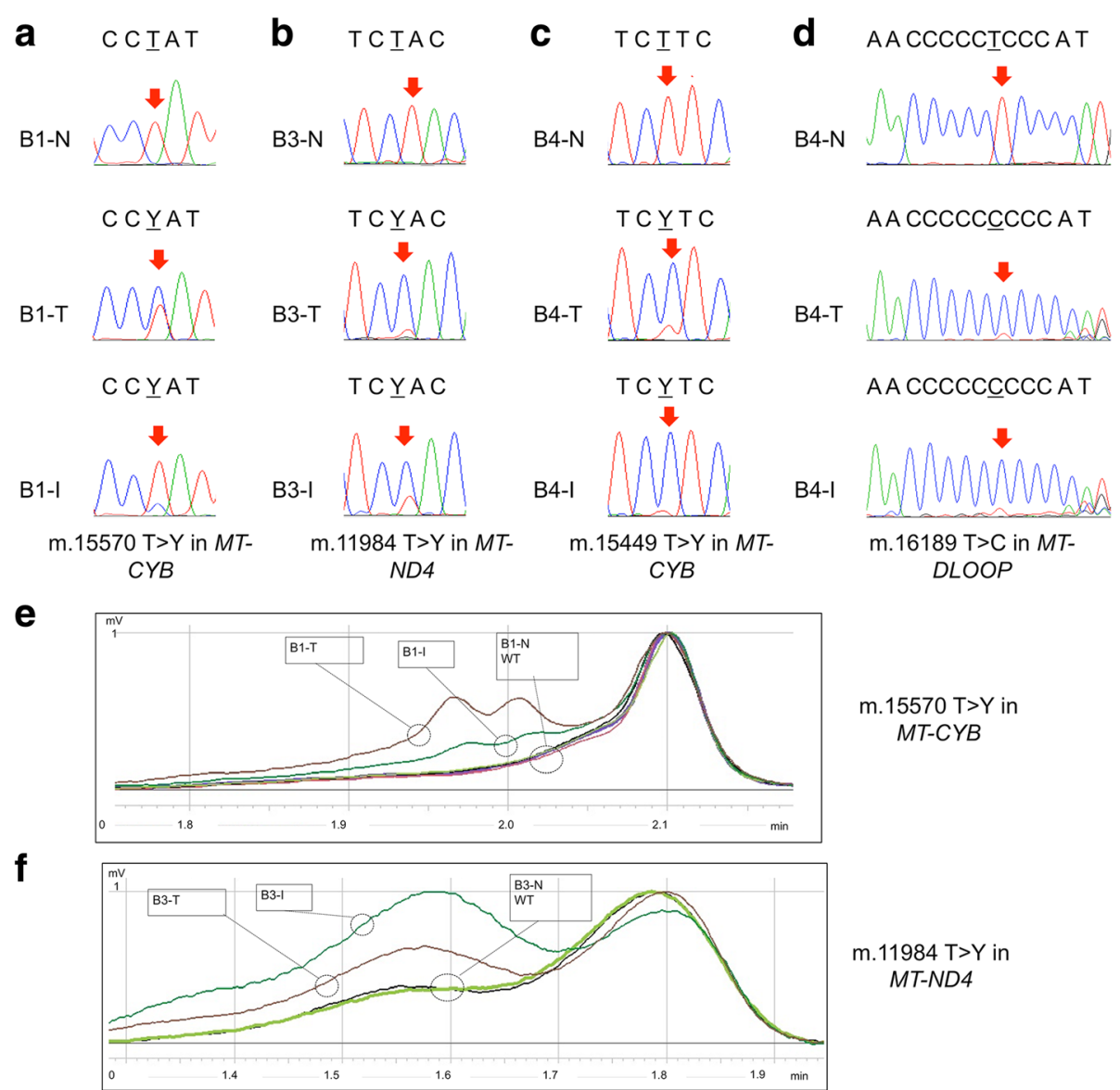

Fig. 1 Mitochondrial DNA sequencing in BOTs and implants $(\mathbf{a}, \mathbf{b}, \mathbf{c}, \mathbf{d})$. Electropherograms of mitochondrial loci harboring mutations in BOT and peritoneal implant samples. Red arrows indicate the mutated bases. (e, f) Representative $\mathrm{AHPLC}$ elution profiles for the in-depth investigation of the somatic nature of mitochondrial DNA mutations m.15570 T > Y/MT-CYB in case B1 and m.11984 T> Y/MT-ND4 in case B3. Homo- and hetero-duplexes are distinguished based on different retention times. (e) Two elution curves for T (Tumor) and I (Implant) (heteroduplex and homoduplex) and a single elution curve for non-tumor tissue $(\mathrm{N})$ and three wild-type controls are present in the analysis of m.15570 T > Y/MT-CYB in case B1. Wild-type (black, pink and purple), N (light green), I (green), T (brown). (f) Two elution curves for T and I (heteroduplex and homoduplex) and a single elution curve for non-tumor tissue ( $\mathrm{N}$ ) and one wild-type control are present in the analysis of m.11984 T>Y/MT-ND4 in case B3. Wild-type (black), N (light green), I (green), T (brown) 
were heteroplasmic in the tissues, although the mutation load may be in such cases underestimated, due to a potential contamination by non-tumor cells, whose complete exclusion from dissected tissues is virtually impossible.

All variants featured a low variability value and, in 4/5 $(80 \%)$ cases, a high score of disease indicating possible candidate variants affecting function (Table 2) [16].

To establish the tumor specificity of the mutations found in both BOTs and implants of the samples B1, B3 and B4 and to confirm a diagnostic efficiency of mitochondrial DNA sequencing we performed dHPLC, which is able to detect heteroplasmic mutations as low as $2 \%$ [18]. Low-level germline heteroplasmy was absent from non-tumor tissue of case B1, in which the m.15570 $\mathrm{T}>\mathrm{C} /(M T-C Y B)$ was shown to be present exclusively in BOT and implant samples (Fig. 1e) and absent from non-tumor tissue. We performed the same analysis for cases B3 (Fig. 1f) and B4 (data not shown) revealing that the $\mathrm{m} .11984 \mathrm{~T}>\mathrm{C} /(M T-N D 4)$ and the $\mathrm{m}$. $15449 \mathrm{~T}>\mathrm{C} /(\mathrm{MT}-\mathrm{CYB})$ were present exclusively in BOT and implant samples and absent from non-tumor tissues. According to these data, the germline nature of mutations found in both BOTs and implant samples of $\mathrm{B} 1, \mathrm{~B} 3$ and $\mathrm{B} 4$ was ruled out, allowing to conclude that tumor-specific mitochondrial DNA mutations in coding genes were detected in $50 \%(4 / 8)$ of BOT at different heteroplasmy levels. In $37.5 \%(3 / 8)$ of the cases, the same mitochondrial DNA mutation was present in both BOT and the peritoneal implant. The extremely low variability of the informative mutations found (Table 2) strengthens the clonality hypothesis, as they are unlikely to occur independently in different cells, as the current variability estimates for mitochondrial genome positions show that a great part of over 16500 nucleotides of the human mitochondrial DNA varies among individuals with different frequency, as it is reported in $\mathrm{HmtDB}$ [17]. It is worth underlining that somatic mutations that were found in our sample set, exclusively in the BOT and were not shared with the peritoneal implant, by no means rule out a clonal origin of the two neoplasms. Mitochondrial DNA variants found only in BOTs may indeed be subsequent to the initial clonal expansion, especially in view of their heteroplasmic nature. It is of note that all somatic mutations in the coding sequences presented a heteroplasmic status in the mass and a very low variability value. They may hence be still unfixed events, particularly since they represent highly damaging genetic lesions. It is known that a certain degree of mitochondrial respiratory chain activity needs to be maintained to progress towards malignancy, and accumulation of damaging mitochondrial DNA mutations blunts tumorigenesis [19]. The occurrence of heteroplasmic mutations may therefore be explained by the need for a metabolic adaptation and by the advantages they confer through the enhancement of reactive oxygen species production associated with tumor promotion [20, 21].

Interestingly, patients B1, B3, B4, and B6 were diagnosed with serous BOTs with noninvasive implants. In sample B3, a small area of low grade intraepithelial carcinoma was found. Patient B1 presented a relapse after the first surgery. Mitochondrial DNA mutations may, in this context, concur to foster transformation of borderline tumor cells into a more aggressive and invasive type of cancer, making their use two-fold both in determining clonality, hence allowing to identify metastases, and to potentially infer a clinical behavior, thus aiding to delineate the prognosis.

We last focused on the variants mapping in the Dloop fragile spots (long C-traits), as these have been already proposed as a marker for clonality [22, 23]. A high frequency of the TC insertion at nucleotide position 310 was found in early stages of serous BOTs [24]. Indeed, we found that the only positions with variants were around nucleotides 303-309, a fragile poly-C stretch termed D310, and 16189, as expected. Inspection of D310 revealed that in patient B6 a heteroplasmic insertion of a cytosine was present only in BOT and peritoneal implant, although Fluorescent PCR [19] revealed a very low load of the insertion in the matched nontumor sample, thereby blunting the informative potential in this case. Sample B5 carried a heteroplasmic insertion of a cytosine in the BOT tissue while peritoneal implant carried the heteroplasmic deletion of two cytosines. The matched non-tumor sample was shown to carry no insertions or deletions. Concerning the analysis of the mitochondrial 16189 variant, characterized by a $\mathrm{T}>\mathrm{C}$ substitution, which produces a highly variable and fragile poly- $\mathrm{C}$ tract, we found the $\mathrm{T}>\mathrm{C}$ substitution in $\mathrm{BOT}$ and implant tissue of patient B4, not occurring in the matched non-tumoral tissue (Fig. 1d), confirming the $\mathrm{BOT}$ and the peritoneal implant to have the same origin.

Overall, tumor-specific mitochondrial DNA mutations were detected in $62.5 \%(5 / 8)$ of BOT at different heteroplasmy levels. In $37.5 \%$ of the patients, the same mitochondrial DNA mutation was present in both the BOT and the peritoneal implant and was therefore informative to infer clonality.

\section{Conclusions}

Besides reporting here for the first time the occurrence of pathogenic mitochondrial DNA mutations in BOTs, our findings have relevant implications in the patient management. Distinguishing between a polyclonal and a monoclonal origin of implants is pivotal in deciding therapeutic options. Indeed, for implants that do not 
originate from the primary tumor a peritoneal carcinogenesis may be envisioned as a potential cause, likely to have occurred within the Müllerian islands, whereby a synchronous tumor to the primary BOT may develop. In this case, removal of the primary BOT does not protect from the formation of implants, implying that a surgical exploration of all peritoneal surfaces and multiple peritoneal biopsies should be mandatory and radical surgery on reproductive organs such as contralateral ovary and uterus plays a secondary role. On the other hand, the demonstration that implants are of a monoclonal derivation implies that they ought to originate from a plundering of the primary tumor. Therefore, in such cases, early diagnosis and removal of the primary tumor becomes pivotal in the prevention of spread within the abdominal cavity, accounting for the importance of molecular analyses capable of providing such a relevant proof of concept.

\section{Additional files}

Additional file 1: Supplementary materials and methods. Detailed Materials and Methods are reported. (DOCX $32 \mathrm{~kb}$ )

Additional file 2: Sample IDs of Borderline Ovarian Tumors, Peritoneal Implants and Non-Tumor tissue. Sequences of B1-B8 samples were submitted to the public database HmtDB (http://www.hmtdb.uniba.it), list of specimens and $\mathrm{HmtDB}$ identifiers are reported. Abbreviations: Borderline ovarian tumors (B); peritoneal implants (I); non-tumor tissue (N). (XLSX 9 kb)

\section{Abbreviations}

BOT: Borderline ovarian tumors; dHPLC: Denaturing High Performance Liquid Chromatography

\section{Acknowledgements}

Not applicable

\section{Funding}

This work was partly supported by Associazione Italiana Ricerca sul Cancro (AIRC) grant IG 14242 - JANEUTICS to G.Gasparre and AIRC grant IG 17387 TOUCHME to A.M.Porcelli; G. Girolimetti is supported by an AIRC triennial fellowship "Livia Perotti".

\section{Availability of data and materials}

Sequences of B1-B8 samples were submitted to the public database HmtDB (http://www.hmtdb.uniba.it), list of specimens and HmtDB identifiers are reported in Additional file 2.

\section{Authors' contributions}

GGa, AMPe and DS conceived the study. GGa, GGi, AMPe and AMPo contributed to the design of the experiments. GGi, LBA and IK carried out the experiments. MP, RP, GD, CC, GC and PDI contributed to samples and clinical information collection. GGa, GGi, AMPe, IK and AMPo drafted the manuscript. All authors read and approved the final manuscript.

\section{Competing interests}

The authors declare that they have no competing interests.

\section{Consent for publication}

Not applicable.

\section{Ethics approval and consent to participate}

This study was conducted within the framework of the Mitochondria in Progression of Endometrial and Ovarian cancer - MiPEO study, approved by the S. Orsola Hospital ethical committee. Internal review board protocols were followed for collection of samples. The reference number is 107/2011/U/Tess.

\section{Author details}

${ }^{1}$ Department of Medical and Surgical Sciences (DIMEC) - Unit of Medical Genetics, University of Bologna Medical School, Via G. Massarenti 9, 40138 Bologna, Italy. ${ }^{2}$ Department of Obstetrics and Gynecology, Oncologic Gynecology Unit, University Hospital S.Orsola-Malpighi, 40138 Bologna, Italy. ${ }^{3}$ Unit of Pathology, University Hospital S.Orsola-Malpighi, 40138 Bologna, Italy. ${ }^{4}$ Unit of Oncology and Transplant Pathology, University Hospital S.Orsola-Malpighi, 40138 Bologna, Italy. ${ }^{5}$ Department of Experimental, Diagnostic, and Specialty Medicine, University Hospital S.Orsola-Malpighi, 40138 Bologna, Italy. ${ }^{6}$ Department of Pharmacy and Biotechnology, University of Bologna, 40138 Bologna, Italy.

Received: 30 August 2016 Accepted: 17 February 2017

Published online: 27 February 2017

\section{References}

1. Lenhard MS, Mitterer S, Kumper C, Stieber P, Mayr D, Ditsch N, et al. Longterm follow-up after ovarian borderline tumor: relapse and survival in a large patient cohort. Eur J Obstet Gynecol Reprod Biol. 2009;145:189-94.

2. Jones MB. Borderline ovarian tumors: current concepts for prognostic factors and clinical management. Clin Obstet Gynecol. 2006;49:517-25.

3. Manek S, Wells M. Pathology of borderline ovarian tumours. Clin Oncol R Coll Radiol G B. 1999;11:73-7.

4. Menczer J, Chetrit A, Sadetzki S. The effect of hysterectomy on survival of patients with borderline ovarian tumors. Gynecol Oncol. 2012;125:372-5.

5. Lalwani N, Shanbhogue AKP, Vikram R, Nagar A, Jagirdar J, Prasad SR. Current update on borderline ovarian neoplasms. AJR Am J Roentgenol. 2010;194:330-6.

6. Seidman JD, Kurman RJ. Ovarian serous borderline tumors: a critical review of the literature with emphasis on prognostic indicators. Hum Pathol. 2000; 31:539-57.

7. Silva EG, Gershenson DM, Malpica A, Deavers M. The recurrence and the overall survival rates of ovarian serous borderline neoplasms with noninvasive implants is time dependent. Am J Surg Pathol. 2006:30:1367-71.

8. Gershenson DM, Silva EG, Tortolero-Luna G, Levenback C, Morris M, Tornos C. Serous borderline tumors of the ovary with noninvasive peritoneal implants. Cancer. 1998;83:2157-63.

9. Abu-Rustum NR, Sonoda Y. Transperitoneal laparoscopic staging with aortic and pelvic lymph node dissection for gynecologic malignancies. Gynecol Oncol. 2007:104:5-8.

10. Lu KH, Bell DA, Welch WR, Berkowitz RS, Mok SC. Evidence for the multifoca origin of bilateral and advanced human serous borderline ovarian tumors. Cancer Res. 1998:58:2328-30.

11. Sieben NL, Roemen GMJM, Oosting J, Fleuren GJ, van Engeland M, Prat J. Clonal analysis favours a monoclonal origin for serous borderline tumours with peritoneal implants. J Pathol. 2006;210:405-11.

12. Guerra F, Girolimetti G, Perrone AM, Procaccini M, Kurelac I, Ceccarelli C, et al. Mitochondrial DNA genotyping efficiently reveals clonality of synchronous endometrial and ovarian cancers. Mod Pathol Off J U S Can Acad Pathol Inc. 2014;27:1412-20.

13. Guerra F, Kurelac I, Magini P, Cormio A, Santini D, Ceccarelli C, et al. Mitochondrial DNA genotyping reveals synchronous nature of simultaneously detected endometrial and ovarian cancers. Gynecol Oncol. 2011;122:457-8

14. Guerra F, Kurelac I, Cormio A, Zuntini R, Amato LB, Ceccarelli C, et al. Placing mitochondrial DNA mutations within the progression model of type endometrial carcinoma. Hum Mol Genet. 2011;20:2394-405.

15. Calabrese C, Simone D, Diroma MA, Santorsola M, Gutta C, Gasparre G, et al. MToolBox: a highly automated pipeline for heteroplasmy annotation and prioritization analysis of human mitochondrial variants in high-throughput sequencing. Bioinforma Oxf Engl. 2014;30:3115-7.

16. Santorsola M, Calabrese C, Girolimetti G, Diroma MA, Gasparre G, Attimonelli M. A multi-parametric workflow for the prioritization of mitochondrial DNA variants of clinical interest. Hum Genet. 2016;135:121-36.

17. Rubino F, Piredda R, Calabrese FM, Simone D, Lang M, Calabrese C, et al $\mathrm{HmtDB}$, a genomic resource for mitochondrion-based human variability studies. Nucleic Acids Res. 2012:40:D1150-1159.

18. Kurelac I, Lang M, Zuntini R, Calabrese C, Simone D, Vicario S, et al. Searching for a needle in the haystack: comparing six methods to evaluate heteroplasmy in difficult sequence context. Biotechnol Adv. 2012;30:363-71. 
19. Mazurek S, Shoshan MC. Tumor Cell Metabolism Pathways, Regulation and Biology. Wien: u.a.: Springer; 2015

20. Larman TC, DePalma SR, Hadjipanayis AG, Protopopov A, Zhang J, Gabriel $S B$, et al. Spectrum of somatic mitochondrial mutations in five cancers. Proc Natl Acad Sci U S A. 2012;109:14087-91.

21. Park JS, Sharma LK, Li H, Xiang R, Holstein D, Wu J, et al. A heteroplasmic, not homoplasmic, mitochondrial DNA mutation promotes tumorigenesis via alteration in reactive oxygen species generation and apoptosis. Hum Mol Genet. 2009;18:1578-89.

22. Masuda S, Kadowaki T, Kumaki N, Tang X, Tokuda Y, Yoshimura S, et al. Analysis of gene alterations of mitochondrial DNA D-loop regions to determine breast cancer clonality. Br J Cancer. 2012;107:2016-23.

23. Geurts-Giele WRR, Gathier GHGK, Atmodimedjo PN, Dubbink HJ, Dinjens WNM. Mitochondrial D310 mutation as clonal marker for solid tumors. Virchows Arch Int J Pathol. 2015:467:595-602.

24. Aikhionbare FO, Mehrabi S, Kumaresan K, Zavareh M, Olatinwo M, Odunsi K, et al. Mitochondrial DNA sequence variants in epithelial ovarian tumor subtypes and stages. J Carcinog. 2007;6:1.

Submit your next manuscript to BioMed Central and we will help you at every step:

- We accept pre-submission inquiries

- Our selector tool helps you to find the most relevant journal

- We provide round the clock customer support

- Convenient online submission

- Thorough peer review

- Inclusion in PubMed and all major indexing services

- Maximum visibility for your research

Submit your manuscript at www.biomedcentral.com/submit
Biomed Central 